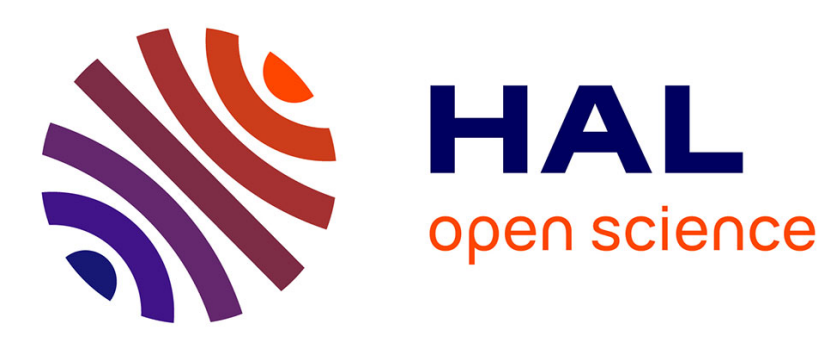

\title{
De la gestion forestière durable à la bonne gouvernance des forêts : la production d'indicateurs, toujours
}

Gérard Buttoud

\section{To cite this version:}

Gérard Buttoud. De la gestion forestière durable à la bonne gouvernance des forêts: la production d'indicateurs, toujours. Revue forestière française, 2012, 64 (5), pp.583-589. 10.4267/2042/50647 . hal-03446880

\author{
HAL Id: hal-03446880 \\ https://hal.science/hal-03446880
}

Submitted on 24 Nov 2021

HAL is a multi-disciplinary open access archive for the deposit and dissemination of scientific research documents, whether they are published or not. The documents may come from teaching and research institutions in France or abroad, or from public or private research centers.
L'archive ouverte pluridisciplinaire HAL, est destinée au dépôt et à la diffusion de documents scientifiques de niveau recherche, publiés ou non, émanant des établissements d'enseignement et de recherche français ou étrangers, des laboratoires publics ou privés. 


\title{
DE LA GESTION FORESTIÈRE DURABLE À LA BONNE GOUVERNANCE DES FORÊTS : LA PRODUCTION D'INDICATEURS, TOUJOURS
}

\author{
GÉRARD ButToud
}

\section{DES CRITÈRES ET INDICATEURS (C\&I) DE GESTION DURABLE : POUR QUOI FAIRE ?}

Pour qui remonte à la genèse du débat international sur le développement durable en forêt, s'impose vite l'idée que les principes, critères, indicateurs et autres vérificateurs de la gestion durable des forêts sont nés de l'impossibilité de tous les acteurs à se mettre immédiatement d'accord sur ce que recouvrait exactement la gestion durable des forêts (GDF). Faute de pouvoir définir le concept autrement qu'en recourant à la rhétorique de la synthèse, habituelle dans les grandes conférences internationales, il fallait, pour espérer avoir un impact précis, définir la GDF par un ensemble de composantes plus ou moins normées qui parleraient aux spécialistes et se déclineraient différemment selon les lieux (Wang, 2004).

Les certificateurs, pressés de remplacer l'État par d'autres mécanismes de décision, s'emparèrent d'abord du concept, s'en servant pour définir les règles d'évaluation de la qualité de la gestion des forêts. La généralisation progressive du principe de certification à un nombre toujours plus grand de forêts a finalement fait des indicateurs l'un des outils majeurs d'appréciation de la qualité de la gestion forestière, sans pour autant que l'on s'en serve - et c'est bien là leur principale limite - pour orienter cette même gestion (Buttoud coord., 2004). S'ils sont devenus des instruments privilégiés d'appréciation de ce qui se fait à l'échelle nationale ou européenne, les indicateurs de gestion durable n'ont guère servi jusqu'ici à orienter les choix et décisions de gestion pris au niveau de la région ou du massif forestier.

\section{LES INDICATEURS DE GESTION DURABLE SERVENT-ILS À ORIENTER LES POLITIQUES ?}

À plus forte raison n'ont-ils donc guère servi en matière de politique forestière.

Les raisons freinant l'utilisation des indicateurs de GDF dans les politiques forestières sont de plusieurs ordres.

D'abord la plupart de ces indicateurs n'ont de sens que si l'on s'y réfère a posteriori. C'est seulement une fois l'action conduite qu'ils permettent d'apprécier si celle-ci s'est faite correctement. Or en politique, plus peut-être qu'en matière de gestion, les décideurs ont surtout besoin de référents leur permettant le plus rapidement possible de corriger l'action, sans attendre qu'elle soit menée à son terme. Tels qu'actuellement conçus, les indicateurs de GDF ne peuvent guère servir à ce suivi de tous les instants. 
Ensuite la nécessité d'associer un nombre de plus en plus élevé d'acteurs différents amène à prendre en compte des avis eux-mêmes différents. Des instruments aussi normés que des indicateurs de GDF ne peuvent traduire cette diversité qui est constituante des politiques en question, l'objectivité en la matière s'avérant pour le moins illusoire.

Enfin, les valeurs intangibles ou culturelles ne sont pratiquement pas représentées dans les listes d'indicateurs de GDF qui sont utilisées, même si les grandes réunions internationales ne cessent de rappeler leur importance. Leur quasi-absence empêche alors de porter un jugement adéquat sur les politiques par le seul usage d'indicateurs usuels de GDF.

À ces limites d'ordre conceptuel, s'en ajoutent d'autres, plus techniques.

D'abord, les indicateurs de GDF servent à décrire des actions propres au secteur forestier, au moment où tous les spécialistes des politiques s'accordent à penser que l'intersectorialité est une caractéristique de base des systèmes de décision publique dans le domaine. L'impact en forêt de mesures environnementales, douanières, fiscales, est souvent plus important que celui de décisions purement forestières. L'utilisation des indicateurs de GDF pour suivre et évaluer les politiques se trouve donc bornée à des considérations sur la forêt qui, pour être importantes, ne sont peut-être pas primordiales, en tous cas pas explicatives.

Enfin, en politique, il est difficile de lier la mesure et l'impact supposé, ce qui limite l'emploi d'indicateurs de résultats. Il suffit de garder en mémoire les débats politiques enflammés opposant des protagonistes qui concluent de façon opposée en utilisant les mêmes chiffres pour être convaincu que les politiques renâclent à se voir appliquer des raisonnements déductifs linéaires.

Finalement, dans les politiques publiques, on s'attache surtout aux grands objectifs et aux lignes stratégiques, au pourquoi et au vers quoi. Le comment importe sans doute, mais ne vient qu'après. Quant aux résultats, bien malin qui peut d'emblée les prévoir (Buttoud ed., 2006). Alors on comprend qu'avec autant de limites mises bout à bout, des réticences se soient fait jour dans l'utilisation des indicateurs de GDF en matière de politique forestière.

La planification stratégique quand elle existe (elle est peu développée en France) fait sans doute une meilleure part aux indicateurs de gestion durable, peut-être parce que la nature technique de ces indicateurs les rapproche des préoccupations des décideurs locaux.

\section{DE QUELS TYPES DE CRITÈRES ET INDICATEURS LES POLITIQUES ONT-ELLES BESOIN ?}

En politique, on a généralement besoin de deux types d'indicateurs.

Les premiers doivent permettre d'apprécier en permanence si la politique est correctement formulée. L'intégration nécessaire d'objectifs différents et parfois concurrents visant la durabilité des points de vue écologique, économique et social, peut se faire selon les cas par optimisation sous contrainte (dans le cas de décisions techniques spécifiques prises par un corps de décideurs techniques spécialisés - c'est encore le cas général, y compris en France), ou bien de plus en plus par négociation avec les acteurs intéressés (et là, le rôle de l'État ne se définit plus de la même façon). Dans ces deux cas, les indicateurs requis se rapportent à la correspondance entre objectifs et moyens pour le premier, et au nombre d'acteurs impliqués dans le second.

Le deuxième type d'indicateurs utiles comprend ceux dont on peut se servir pour vérifier en continu que la politique est correctement appliquée. Dès qu'on en vient à l'application concrète, les mots clés qui reviennent le plus souvent sont ceux d'efficacité (quand on compare les 
résultats effectifs de l'action à ceux qui étaient escomptés à son début), d'efficience (où l'on met en parallèle les moyens et les résultats pour apprécier si l'usage des ressources a été optimal), et de plus en plus de partenariat (quand les actions sont conduites en collaboration par plusieurs acteurs), de transparence (quand ce qui est fait est connu de tous les protagonistes), parfois même de capacité (c'est-à-dire l'appréciation que les acteurs étaient équipés ou non de tous les éléments devant leur permettre de mener à bien les actions conduites).

À l'exception de celui d'efficacité, peu de ces mots clés renvoient à des référents présents dans la définition des indicateurs de GDF. Par contre, on voit à l'énoncé des problématiques sousjacentes, qui mettent l'accent sur les conditions d'application et de suivi des actions conduites, qu'il s'agit autant sinon plus de gouvernance que de politique (cf. Kouplevatskaya-Buttoud, 2009).

\section{Les critères et indicateurs de bonne gouvernance des forêts : vers un nouveau type de suivi des politiques?}

Depuis le début des années 2000 en effet, on parle sans doute moins de gestion forestière durable que de bonne gouvernance des forêts (Cashore et al., 2004; Buttoud coord., 2007 ; Agrawal et al., 2008). C'est que les déclarations et prescriptions internationales se sont vite heurtées à la réticence des faits. Faire de la gestion durable, surtout dans les forêts tropicales qui continuent à cristalliser l'attention des grandes conférences, ce n'est finalement pas si simple.

L'idée a alors germé que si cela ne marchait pas, ce n'était pas nécessairement faute de normes techniques; c'était qu'on s'y prenait mal pour appliquer lesdites politiques, ou même qu'on ne faisait pas grand-chose pour cela. Comme on parlait alors beaucoup de durcir les règles, des spéculateurs peu scrupuleux se dépêchaient de faire n'importe quoi avant d'être plus tard contraints, ce qui augmentait l'exploitation et le commerce illégaux (en tous cas aux marges de la légalité). Ce qu'il fallait donc faire si l'on visait toujours une gestion durable des forêts, c'était de la bonne gouvernance.

Ce nouveau concept était lui-même difficile à définir de façon consensuelle, tant il prêtait à interprétations et appropriations diverses selon les acteurs (EC, 2001 ; UNDP, 2006).

On restait aussi dans le moral, le normatif et souvent même le prescriptif. Comme lorsqu'il s'était agi de gestion durable des forêts, différents organismes et ONG arrivaient aussitôt avec leur propre avis, leur propre définition, leurs propres idées sur ce qu'il y avait lieu de faire.

Ces deux considérations devaient alors conduire à la production d'un nouveau type de C\&I, ceux censés orienter ce qu'on allait appeler la «bonne gouvernance» des forêts.

L'histoire se réécrivait sur un mode semblable à celui des années 1990. Des instituts privés de recherche, plus ou moins liés à de grandes organisations non gouvernementales de promotion de la démocratie, cherchaient à établir une liste de composants de cette fameuse gouvernance : l'Institut pour les ressources mondiales (WRI, 2009), des ONG comme Chatham House (Saunders et Reeve, 2010) et Transparency International proposaient leurs schémas de pensée, d'ailleurs très proches.

La Banque mondiale qui parmi les premiers avait lancé le concept de gouvernance, qu'elle définissait comme une autre forme de politique où l'État négociait les grands choix avec les forces sociales et économiques et suivait en continu ce qui se faisait, devait conserver l'initiative de regrouper ces approches dans une démarche globale commune (Kauffman et Kraay, 2008 ; Gareth, 2011). En 2010, elle réussissait à tirer la FAO pour élaborer, avec les ONG et les scientifiques de l'Institut forestier européen (EFI) et du Centre international de recherche sur les forêts (CIFOR), 
une liste de C\&I de bonne gouvernance applicables à l'ensemble des forêts (pas seulement tropicales) de la planète, diffusés en 2011 dans un contexte fortement dépendant des choix internationaux en matière de lutte contre le réchauffement global (Profor-FAO, 2011).

\section{LE MODÈLE INTERNATIONAL D’ÉVALUATION DE LA QUALITÉ DE LA GOUVERNANCE}

Le cadre général établi énumère six principes - l'équivalent de critères - (responsabilité des décideurs publics vis-à-vis de la société - pour accountability -, effectivité, efficience, participation, équité, transparence) correspondant au discours international sur la démocratie, laquelle est ici implicitement supposée garante de la durabilité. Ces six critères sont appliqués à trois piliers des politiques publiques (cadre politique, réglementaire et institutionnel; planification et processus de décision publique; application, suivi et contrôle). L'analyse des problématiques globales du secteur forestier amène alors à identifier à l'intérieur de ces piliers treize composants (cf. tableau I, ci-dessous) décomposés eux-mêmes en soixante-dix-huit sous-composants, parmi lesquels les indicateurs les plus appropriés à répondre aux questions politiques posées seraient choisis par les décideurs (États, bailleurs de fonds), en articulation (Secco et al., 2011) ou non aux schémas de certification. Il n'y a donc pas d'indicateurs préétablis, et qui seraient tous mesurés.

TABLEAU I Structure du cadre international d'évaluation de la qualité de la gouvernance

(d'après Profor-FAO, 2011)

Pilier 1 : Cadre politique, réglementaire et institutionnel

11. Politiques et législations relatives à la forêt

12. Cadre légal d'appui à la gestion foncière, propriété et droits d'usage

13. Concordance des politiques publiques générales ou d'autres secteurs avec les politiques forestières

14. Cadres institutionnels

15. Incitations financières, mécanismes économiques et partage des bénéfices

Pilier 2 : Planification et processus de décision publique

21. Participation des parties prenantes

22. Transparence et crédibilité

23. Capacité et activités des parties prenantes

Pilier 3 : Application, suivi et contrôle

31. Administration et gestion des ressources forestières

32. Suivi de l'application des lois forestières

33. Administration et gestion des droits fonciers et de propriété

34. Coopération et coordination

35. Mesures pour lutter contre la corruption

Chaque sous-composant identifié dans le cadre peut servir de base à un ou plusieurs indicateurs selon le problème posé et la précision recherchée. Les indicateurs qui en résultent sont tous de nature qualitative, et mesurent le niveau de qualité de la gouvernance au moyen d'une grille de valeur définie de façon collective en début d'exercice (par exemple quatre niveaux, de zéro à trois, si l'on veut mesurer comment l'administration s'appuie sur des données statistiques et d'inventaires adéquates : 0 : aucune information de ce type n'existe ; 1 : ces informations existent partiellement et sont peu utilisées ; 2 : ces informations existent et sont utilisées au cas par cas ; 3 : ces informations sont complètes, et systématiquement actualisées et utilisées). 
Cette approche, adaptée à la globalisation politique et reposant sur des critères moraux, doit dès lors instaurer une nouvelle façon d'évaluer, et donc peut-être aussi d'orienter, les politiques nationales forestières. L'une de ses innovations les plus marquantes consiste en tous cas à considérer qu'il n'y a pas de "bonne " ou de "mauvaise " gouvernance, mais une "plus ou moins bonne » gouvernance qui résulterait de cette analyse détaillée et composite.

\section{POUR UNE INTÉGRATION ENTRE C\&I DE GESTION DURABLE ET DE BONNE GOUVERNANCE : L'AVENIR ?}

En quoi ces nouveaux C\&l de bonne gouvernance sont-ils semblables et différents de ceux anciennement définis pour évaluer (et peut-être aussi guider) la gestion durable des forêts ? Certes les deux approches ne sont pas neutres, dans la mesure où la première, celle des indicateurs de GDF, sert surtout à contrôler les résultats de la gestion, et la seconde, celle des indicateurs de gouvernance, répond au souci de ceux (bailleurs de fonds, institutions nationales de contrôle de l'action publique) souhaitant apprécier la façon avec laquelle la politique est conduite. La première s'avère surtout utile aux niveaux régional et local, par exemple dans le cas de plans régionaux intégrés, alors que la seconde trouve sa logique aux plans national et mondial. L'application des C\&I de GDF ne répond qu'indirectement aux questions liées à l'application des politiques, mais peut servir à améliorer le contenu de celles-ci, alors que les indicateurs de gouvernance renseignent sur le comment, mais pas sur le contenu, de ces mêmes politiques.

Plus qu'opposées, elles apparaissent ainsi complémentaires. L'approche par les indicateurs de GDF peut très bien être appliquée de telle sorte que de nouveaux indicateurs, plus appropriés à évaluer la qualité de la gouvernance, soient ajoutés d'un commun accord à la liste de référence. De même l'approche par les indicateurs de gouvernance peut - encore plus facilement puisqu'il n'y a pas de liste préconçue définitive - intégrer des indicateurs de durabilité (par exemple sur l'efficacité de l'administration, des indicateurs comme le niveau d'application du programme de l'administration, le niveau d'efficacité du programme de l'administration, le niveau d'impact de l'administration en matière de gestion durable, peuvent être mesurés).

En fait, il y a un grand intérêt à croiser les différents indicateurs employés dans ces deux types d'approche.

Des indicateurs de GDF, redéfinis au cas par cas en fonction des problématiques du lieu et du moment, peuvent permettre d'abord de mieux bâtir et suivre les politiques. Plusieurs tentatives ont été conduites, souvent par des chercheurs et souvent aussi de façon empirique, pour répondre aux questions auxquelles les politiques étaient confrontées (par exemple Cornet et Rajapbaev, 2004). C'est l'objet même de la méthode dite TOSIA (acronyme anglais de Tool for Sustainability Impact Assessment), basée sur des indicateurs spécifiques définis localement et permettant de prendre en compte les valeurs immatérielles aussi bien que matérielles par une étude d'impact ex ante en matière de durabilité comparant l'incidence de décisions alternatives, même publiques, en matière écologique, économique et sociale.

Une telle approche permet la formulation d'indicateurs qualitatifs qu'on peut croiser avec ceux prônés en matière de gouvernance. Pour peu que le cadre conceptuel alternatif proposé par les agences internationales soit analytique et non prescriptif - ce qui est annoncé mais reste quand même à vérifier -, cet ensemble composite d'indicateurs de qualité des politiques peut servir de base à la construction d'une séquence d'apprentissage collectif de ce que sont à la fois la durabilité et la gouvernance (Buttoud ed., 2011). L'intégration d'indicateurs de GDF et de gouver- 
nance prend alors tout son sens dans le cas de processus de décision participatifs, aux plans aussi bien global (formulation des grandes orientations politiques) que local (exemple des chartes forestières de territoire). Ce n'est pas son moindre intérêt.

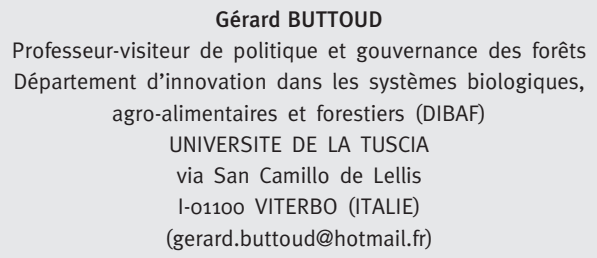

\section{BIBLIOGRAPHIE}

AGRAWAL (A.), CHHATRE (A.), HARDIN (R.). - Changing governance of the world's forests. - Science, $\mathrm{n}^{\circ} 320$, 2008, pp. 1460-1462.

BUTTOUD (G.) ed. - Change in forest governance as a collective learning process. - Forest Policy and Economics, vol. 13 , issue 2, 2011, pp. 87-154.

BUTTOUD (G.) coord. - Critères et indicateurs de gestion durable des forêts : où en est-on ? - Revue forestière française, vol. LVI, $\mathrm{n}^{\circ} 5,2004$, pp. 361-502.

BUTTOUD (G.) ed. - The evaluation of forest policies and programmes. - Forest Policy and Economics, vol. 8, issue 5, 2006, pp. 499-578.

BUTTOUD (G.) coord. - Nouvelles approches de la gestion et de la gouvernance forestières. - Revue forestière française, vol. LIX, $\mathrm{n}^{\circ} 5$, 2007, pp. 433-576.

CASHORE (B.), AULD (G.), NEWSON (D.). - Governing through markets: forest certification and the emergence of non-State authority. - New Haven : Yale University Press, 2004.

CORNET (J.G.), RAJAPBAEV (M.). - Criteria and indicators of sustainable management of Juniper forests in South-Kyrgyzstan. - Nancy : ENGREF - Laboratoire de Politique forestière, 2004.

EUROPEAN COMMISSION. - European governance; a white paper. - Brussels : European Commission, 2001.

GARETH (W.). - What makes a good governance indicator? - Policy Practice brief $\mathrm{n}^{\circ}$ 6. - Brighton : The Policy Practice Ltd, 2011.

KAUFFMAN (D.), KRAAY (A.). - Governance indicators: where are we, where should we be going? - The World Bank Research Observer, vol. 23, $\mathrm{n}^{\circ}$ 1, 2008, pp. 1-30.

KOUPLEVATSKAYA-BUTTOUD (I.). - Adaptation to change and re-designing of governance systems: cases from small-scale rural forestry. - Small-scale Forestry, vol. 8, $\mathrm{n}^{\circ}$ 2, 2009, pp. 231-247.

PROFOR-FAO. - Framework for assessing and monitoring forest governance. - Rome : The Program on Forests - Food and Agriculture Organisation of the United Nations, 2011.

SAUNDERS (J.), REEVE (R.). - Monitoring governance for implementation of REDD+. - London : Chatham House, 2010.

SECCO (L.), TASSA (D.), DA RE (R.), GATTO (P.). - How to measure governance in forestry: key-dimensions and indicators from emerging economic mechanisms. - German Journal of Forest Research, vol. 182, $\mathrm{n}^{\circ}$ 5/6, 2011, pp. 69-82.

UNDP. - Governance indicators; a users' handbook. - New York : United Nations Development Programme, 2006.

WANG (S.). - One hundred faces of sustainable forest management. - Forest Policy and Economics, $\mathrm{n}^{\circ} 6$, 2004, pp. 205-213.

WRI. - The governance of forests tool-kit; a draft framework of indicators for assessing governance of the forest sector. - Washington DC: The World Resources Institute, 2009. 
DE LA GESTION FORESTIËHE DURABLE A LA BONNE GOUUERNANCE DES FORÊTS : LR PAODUCTION D'INDICATEURS, TOUJOURS [Hésumé]

Les critères et indicateurs (C\&l) de gestion durable des forêts (GDF), conçus pour aider à mieux cerner ce qu'est justement la GDF, n'ont fourni jusqu'ici qu'un apport limité à l'orientation des politiques publiques sur les forêts. Peu employés pour l'évaluation de ces politiques, ils ont d'abord servi au contrôle de la gestion, et parfois donné un argumentaire, au moins rhétorique, aux bailleurs de fonds et décideurs internationaux.

S'agissant de la politique forestière, la difficulté d'utiliser les indicateurs de résultats a progressivement conduit à privilégier les indicateurs de gouvernance, sur lesquels les agences internationales de développement - Banque mondiale, Organisation des Nations unies pour l'alimentation et l'agriculture (FAO) viennent de s'entendre avec des organisations non gouvernementales (ONG) internationales. Formée essentiellement d'indicateurs de système, se voulant moins prescriptive, cette nouvelle perspective s'articule plus ou moins avec la recherche systématique de la GDF.

L'analyse des limites politiques des C\&I de gestion durable des forêts n'enlève cependant rien à leur rôle de support possible à la démocratisation des décisions publiques dans le secteur forestier, dans la mesure où ils servent au mécanisme d'apprentissage entre acteurs dans la construction de ce qu'est la gestion et la politique des forêts. Ils prennent tout leur sens dans le cas de processus de décision participatifs, aux plans aussi bien global (formulation des grandes orientations politiques) que local (exemple des chartes forestières de territoire).

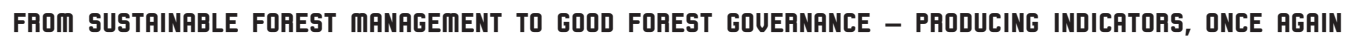
[Abstract]

Criteria and indicators (C\&I) of sustainable forest management (SFM) have been designed with the aim of defining better what SFM consists in, but they have provided limited help in guiding public policies on forests. They are seldom used for evaluation of those policies, and have mainly contributed to the control of management practices, sometimes providing arguments, albeit rhetorical, to international donors and decisionmakers.

The difficulty in using outcome indicators in forest policy evaluation has progressively led to putting greater emphasis on indicators of governance, which the international development agencies - the World Bank, the Food and Agriculture Organization of the United Nations (FAO) - have just agreed upon with non governmental organisations. Made up essentially of organisational indicators that are intended to be less prescriptive, this new perspective links in, to varying degrees, with systematic targeting of SFM.

The limitations of C\&I for SFM in terms of policy development does not in any way prevent them from playing a role as a potential basis for democratization of public decisions in the forest sector, to the extent that they serve as a learning mechanism among actors in building up forest management and policy. This is particularly meaningful in the case of participatory decision processes, at both global (formulation of policy guidelines) and local (example of the forest territories charters) levels. 
